\title{
Medico-legal Aspects of Traumatic Injury of the Vertebrobasilar Artery
}

\author{
Kouichi HIRAIWA, Taku SATO*, Tatsuya SASAKI*, Ikubumi MIZUSAWA, \\ Masayuki NATA**, and Namio KODAMA*
}

Departments of Legal Medicine and *Neurosurgery, Fukushima Medical University, Fukushima;

** Department of Forensic Medicine and Sciences, Mie University School of Medicine, Tsu, Mie

\begin{abstract}
Based on our experiences with medico-legal autopsies, we analyzed traumatic injury of the vertebrobasilar artery (VBA) in traffic accident victims. VBA rupture occurred in six cases with traumatic medullary lesions, but in none with cervical cord injury. A relatively small external force applied to the head or neck can induce isolated traumatic subarachnoid hemorrhage (SAH), which accounts for approximately $11 \%$ of fatal head injuries. We examined the relationship between site of impact and site of vascular injury in 16 cases of isolated traumatic SAH. The results showed that the vertebral artery (VA) on the side of impact tended to be longitudinally injured in victims experiencing an external force equivalent to that of a fist punch to the head or face. There was no clear relationship between the site of impact and the site of vascular injury in victims who had sustained an external force greater than that of a fist punch. However, our results suggest that an external force causing rotation of the head was likely to cause tearing of the artery. In cases in which an external force equivalent to that of a fist punch was applied to the head, a raised blood alcohol level was a significant risk factor for artery rupture. Furthermore, a difference in the diameters of the left and right VAs was a risk factor for artery rupture. To determine why the VA, the most frequent site of arterial dissection, is also frequently the site of injury, we histologically examined normal and dissected VBAs. We also discuss medico-legal issues of the causal relationship between external force applied and rupture or dissection of the VA.
\end{abstract}

Key words: traumatic injury, vertebral artery, basilar artery, subarachnoid hemorrhage, legal medicine

\section{Introduction}

Traffic accident victims frequently present with traumatic injury of the vertebrobasilar artery (VBA), an injury not frequently encountered by neurosurgeons because of the high mortality. We reviewed results of legal autopsies of traffic accident victims who died of traumatic brainstem lesions. An analysis of the relationship between their traumatic brainstem lesions and traumatic VBA rupture indicated the possible occurrence mechanism. ${ }^{5)}$

We also reviewed results of legal autopsy cases with isolated traumatic subarachnoid hemorrhage (SAH). A relatively small external force applied to the head or neck caused VBA rupture, resulting in isolated traumatic SAH without brain damage. ${ }^{7}$ To investigate the reasons for the high incidence of injury to the vertebral artery (VA), the most frequent site of dissecting aneurysms, we histologically examined normal and dissected VBAs. ${ }^{12}$ ) Based on the results, we discuss the medico-legal issues of the causal relationship between external force applied and rupture or dissection of the VA.

\section{Traumatic Brainstem Lesions and VBA Injury}

Traumatic brainstem tear, first described by Lindenberg and Freytag, ${ }^{10}$ was initially regarded as an injury due to hyperextension of the head. Brainstem lesions are now recognized as sequelae rather than direct results of trauma. ${ }^{1)}$ One of the most widely accepted mechanisms of pathogenesis is hyperextension of the neck due to face or forehead impact. ${ }^{10)}$ Brainstem injuries accompanied by ring fractures of the base of the skull were produced by not only hyperextension or anteflexion but also by torsion or other forces applied to the head. ${ }^{15)}$ Brainstem lesions are often seen in victims of high-speed car accidents whose face or forehead struck the 
dashboard or windshield frame..$^{3,10,14,15)}$ Nine victims of traffic accidents, including eight pedestrians, sustained brainstem and/or upper cervical cord lesions. ${ }^{9)}$ We also found brainstem lesions primarily in pedestrians involved in traffic accidents.

We reviewed 156 traffic accident deaths that occurred between 1985 and 2000 to determine the morbidity and pathological features of traumatic brainstem lesions as well as to assess the relationship between these lesions and the applied force. ${ }^{5)}$ Twenty-six (16.7\%) of the 156 autopsied traffic accident victims sustained gross primary brainstem injury. These 26 victims included 19 pedestrians, five bicyclists, one automobile driver, and one passenger. In four of the victims with upper face impact and two of the victims with lower face impact, the direct force to the head resulted in neck hyperextension. Among the victims with brainstem lesions $(n=26)$, there were significantly more individuals older than 40 years, more pedestrians, and more males positive for increased blood alcohol levels than in the control group $(n=130)$. Males predominated among victims in their 4th and 5th decades of life, but there was no sex difference in victims older than 70 years. Fourteen (74\%) of the 19 pedestrians had been hit from behind, including the right- and left-rear sides. In our series, there was a high incidence of traumatic brainstem lesions not only in automobile drivers and passengers but also in males with elevated blood alcohol levels and in elderly pedestrians hit from behind by a vehicle. Our results showed the possibility that traction force attributed to head inertia is crucial in producing brainstem lesions in pedestrians hit by a vehicle traveling at high speed.

Of 16 victims with medullary lesions, eight had atlanto-occipital dislocations/transections and three had C1-2 forward dislocations/transections. The types of brainstem lesion were pontomedullary avulsion in two cases, pontomedullary tears in four cases, and lower medulla avulsion in two cases among the eight cases of atlanto-occipital dislocations/transections, and pontomedullary avulsion in one case, pontomedullary tear in one case, and lower pons avulsion in one case among the three cases of C1-2 forward dislocations/transections. All seven victims with cervical cord lesions had high cervical dislocations/transections that corresponded to the level of their cord lesion.

VBA rupture occurred in six of the 16 victims with medullary lesions. There were no cases with cervical cord lesions. Basilar artery (BA) rupture was seen in six victims, one of whom sustained bilateral VA rupture. None of the victims with brainstem lesions suffered VA rupture without BA rupture.

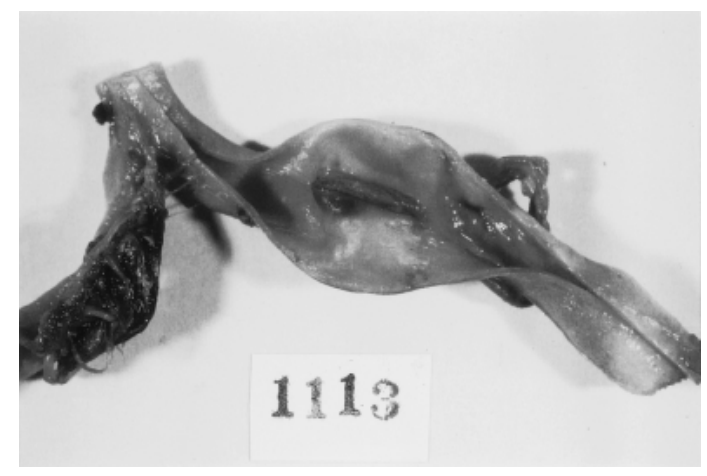

Fig. 1 Photograph showing site of rupture of the right vertebral artery.

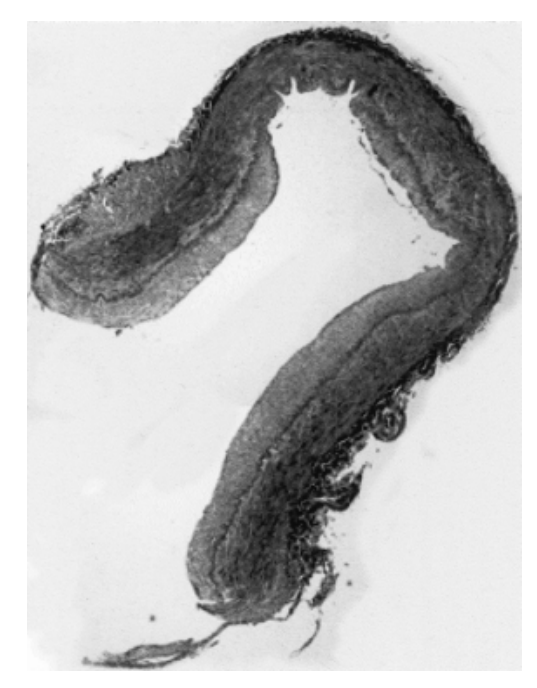

Fig. 2 Photomicrograph showing disruption of the vertebral artery. Modified Masson's trichrome staining for elastic fibers, $\times \mathbf{4 0}$.

Five of the six victims who suffered VBA rupture had atlanto-occipital dislocations/transections and one had C1-2 forward dislocations/transections.

VBA rupture was accompanied by medullary lesion in $38 \%$ of cases. VBA rupture was rarely seen in cases in which the brainstem was torn or avulsed. The VBA had stronger compliance than the brainstem, and the VA had stronger compliance than the BA.

\section{Isolated Traumatic SAH}

In the field of legal medicine, the possible induction of SAH by a relatively small external force applied to the head or neck is known as isolated traumatic $\mathrm{SAH}$, and isolated traumatic SAH is mainly due to VBA rupture (Figs. 1 and 2). ${ }^{2,4,11,17)}$ However, it is not 


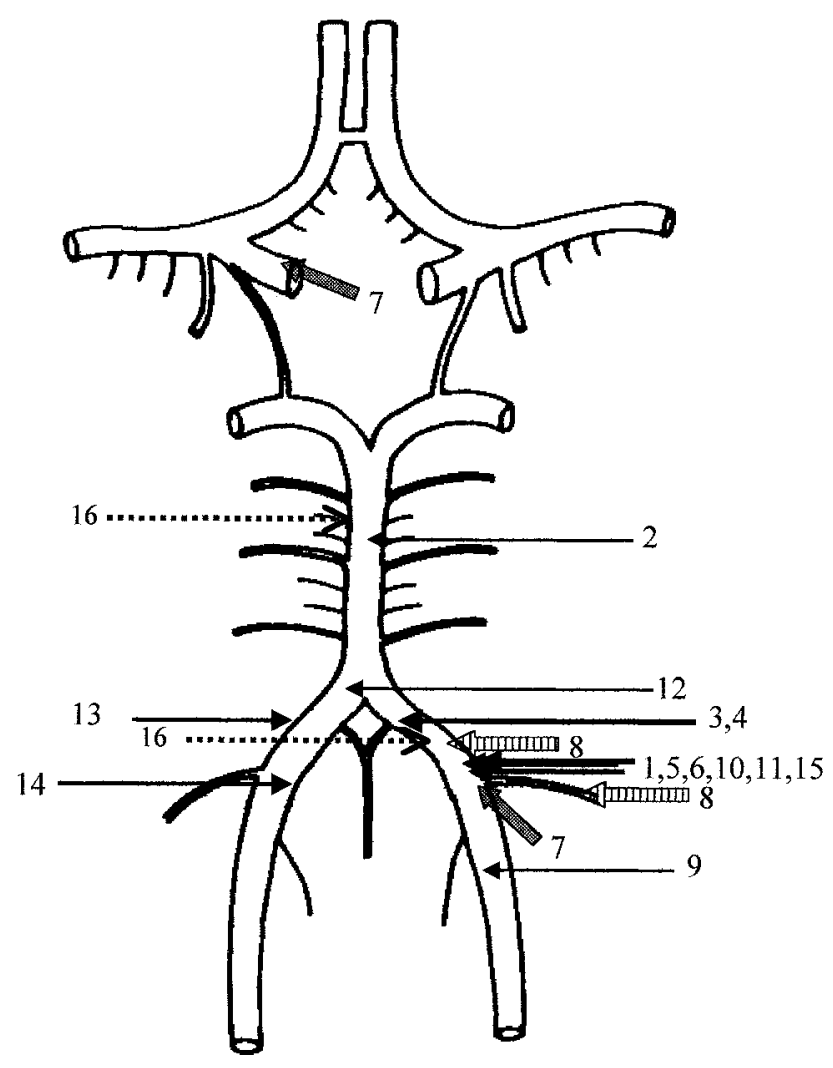

Fig. 3 Distribution of cerebral artery ruptures and dissections. Arrowheads show the direction of head movement. Left and right arrows indicate rightward and leftward rotations of the head, respectively.

clear whether external or internal factors are responsible for the mechanisms as well as the artery dissection that sometimes occurs suddenly in the region of the VBA. Artery rupture is a more severe injury than artery dissection. We speculated that analysis of cases of isolated traumatic SAH could shed light on these mechanisms. We therefore examined fatalities due to isolated traumatic $\mathrm{SAH}^{7)}$ among 1,008 medico-legal autopsies performed at Fukushima Medical University between 1983 and 1999. In $169(17 \%)$ of these 1,008 cases, death was due to head injury, and $14(8.3 \%)$ of the 169 deaths were attributed to isolated traumatic SAH. Excluding the 38 victims whose head was run over by a vehicle, isolated traumatic SAH accounted for $11 \%$ (14/131) of fatal head injuries.

We investigated the relationship between site of impact and site of vascular injury in 16 cases, including 12 cases with autopsies performed at Fukushima Medical University and four cases with autopsies performed in the Division of Forensic Medicine, Tohoku University. We found that the VA on the side of impact tended to be longitudinally injured in victims who had been subjected to an external force equivalent to that of a fist punch to the head or face (Fig. 3). Although there was no clear correlation between the impact site and the site of vascular injury in cases in which the external force was greater than that of a fist punch, our results suggest that an external force causing head rotation could lead to tearing of the artery. We confirmed that the blood alcohol level at the time of application of an external force equivalent to that of a fist punch to the head or face is a significant factor in the likelihood of artery rupture. Another risk factor for arterial rupture is a difference in the diameters of the left and right VAs (Table 1).

The review article by Haldeman et al. ${ }^{6}$ regarding VBA dissections or occlusions is instructive. They reported that dissection was spontaneous in $43 \%$ of their cases and due to cervical manipulation in $31 \%$, trivial trauma in $16 \%$, and major trauma in $10 \%$. Rotation is the most common cervical spinal manipulation procedure used by chiropractors, and the largest number of dissections would be expected to occur after rotational manipulation. These findings agree with our results showing that an external force causing head rotation could lead to tearing of the artery.

We histologically examined normal VAs to determine why the VA is frequently the site of rupture and dissection.

\section{Histology of the Normal VBA}

Although the VA is the most common site of dissecting aneurysms, ${ }^{18)}$ there have been few studies on the histology of the normal VA to clarify the underlying etiologic mechanisms. ${ }^{8,16)}$ We therefore studied the presence, absence, and distribution of the internal elastic lamina (IEL), and the condition of the intima at the site of IEL defects. ${ }^{12)}$

Materials were obtained from 10 autopsy cases, and 20 VAs were examined histologically. The excised VA from the extradural to the intracranial segment was cut at $5-\mathrm{mm}$ intervals, and each segment was fixed in formalin and embedded in paraffin. Twenty serial sections, $4 \mu \mathrm{m}$ in thickness, were prepared from each segment, stained for elastic fibers by elastica Masson stain, and examined under a light microscope.

IEL defects (Fig. 4) were observed at 35 sites in 11 vessels from six subjects aged 51 years or older, but none in the four subjects aged 35 years or younger. In the normal VAs, IEL defects were frequently observed in the extradural portion and near the division of the posterior inferior cerebellar artery 
Table 1 Profiles of 16 fatal cases of isolated traumatic subarachnoid hemorrhage

\begin{tabular}{|c|c|c|c|c|c|c|c|c|c|c|}
\hline $\begin{array}{l}\text { Case } \\
\text { No. }\end{array}$ & Age & Sex & Site of impact & $\begin{array}{l}\text { Cause of } \\
\text { impact }\end{array}$ & $\begin{array}{l}\text { Injured } \\
\text { artery }\end{array}$ & $\begin{array}{l}\text { Length of } \\
\text { injury } \\
\text { (mm) }\end{array}$ & $\begin{array}{l}\text { Shape of } \\
\text { injury }\end{array}$ & $\begin{array}{c}\text { VA } \\
\text { dominancy }\end{array}$ & $\begin{array}{l}\text { Arterio- } \\
\text { sclerotic } \\
\text { change }\end{array}$ & $\begin{array}{c}\text { Drink- } \\
\text { ing }\end{array}$ \\
\hline 1 & 19 & $\mathrm{M}$ & $\begin{array}{l}\text { lt nape } \\
\text { rt nape } \\
\text { rt orbitae }\end{array}$ & $\begin{array}{l}\text { kick } \\
\text { kick }\end{array}$ & lt VA & 12 & longitudinal & no & no & + \\
\hline 2 & 21 & $\mathrm{~F}$ & $\begin{array}{l}\text { lt nape } \\
\text { rt parietooccipital } \\
\text { lt nape }\end{array}$ & $\begin{array}{l}\text { punch } \\
\text { fall down } \\
\text { punch }\end{array}$ & BA & NA & transection & lt & no & + \\
\hline 4 & 29 & M & $\begin{array}{l}\text { lt mastoid process } \\
\text { lt cheek }\end{array}$ & $\begin{array}{l}\text { punch } \\
\text { fall down? }\end{array}$ & lt VA & 5 & longitudinal & lt & no & + \\
\hline 5 & 40 & M & $\begin{array}{l}\text { lt temporal } \\
\text { lt cheek }\end{array}$ & $\begin{array}{l}\text { punch } \\
\text { punch }\end{array}$ & lt VA & 7 & longitudinal & no & no & + \\
\hline 6 & 43 & M & $\begin{array}{l}\text { lt temporal } \\
\text { lt nape }\end{array}$ & $\begin{array}{l}\text { punch } \\
\text { punch }\end{array}$ & $\begin{array}{l}\text { lt VA } \\
\text { lt VA }\end{array}$ & $\begin{array}{l}11 \\
10\end{array}$ & $\begin{array}{l}\text { longitudinal } \\
\text { longitudinal }\end{array}$ & lt & no & + \\
\hline 7 & 48 & M & $\begin{array}{l}\text { rt orbitae } \\
\text { rt cheek }\end{array}$ & $\begin{array}{l}\text { punch? } \\
\text { punch? }\end{array}$ & rt ICA & NA & bulging & lt & no & + \\
\hline 10 & 55 & M & $\begin{array}{l}\text { lt cheek } \\
\text { rt metopic }\end{array}$ & $\begin{array}{l}\text { fall down? } \\
\text { fall down? }\end{array}$ & lt VA & NA & transection & no & no & + \\
\hline 11 & 57 & M & mouth & $?$ & lt VA & NA & longitudinal & no & no & + \\
\hline 12 & 62 & M & $\begin{array}{l}\text { lt cheek } \\
\text { mouth } \\
\text { rt temporal }\end{array}$ & $\begin{array}{l}\text { punch } \\
\text { punch } \\
\text { scoop }\end{array}$ & rt VA & 6 & transection & lt & moderate & + \\
\hline 13 & 48 & $\mathrm{M}$ & $\begin{array}{l}\text { rt metopic } \\
\text { lt parietooccipital } \\
\text { rt parietooccipital }\end{array}$ & $\begin{array}{l}\text { scoop } \\
\text { scoop } \\
\text { iron pipe }\end{array}$ & rt VA & 5 & longitudinal & lt & moderate & + \\
\hline 14 & 59 & $\mathrm{M}$ & $\begin{array}{l}\text { mouth } \\
\text { rt nape }\end{array}$ & $\begin{array}{l}\text { iron pipe } \\
\text { iron pipe }\end{array}$ & rt VA & NA & longitudinal & $\mathrm{rt}$ & no & - \\
\hline 15 & 62 & M & lt nape & timber & lt VA & 5 & longitudinal & no & no & - \\
\hline
\end{tabular}

BA: basilar artery, ICA: internal carotid artery, NA: not available, PICA: posterior inferior cerebellar artery, VA: vertebral artery.

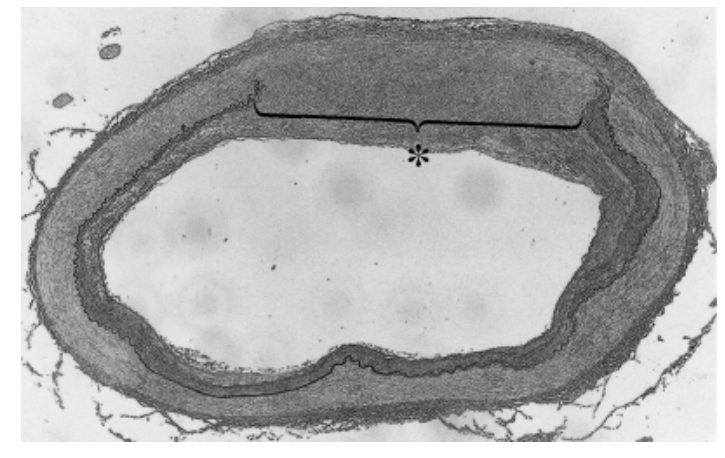

Fig. 4 Photomicrograph showing a defect (asterisk) in the internal elastic lamina of the vertebral artery. Modified Masson's trichrome staining for elastic fibers, $\times \mathbf{4 0}$.

(PICA), areas that are frequent sites of clinical arterial dissection.

Since we were not sure whether IEL defects were specific to the VA, we also examined the BA. Materials were obtained from 21 autopsy cases without organic brain disease. A 2-year-old boy who had drowned had an IEL defect at the basilar bifurcation, and a 55-year-old man had an IEL defect at two sites near the VA junction. Overall, we detected 32 IEL defects in 21 normal BAs, which is similar to the rate reported by us for normal VAs. ${ }^{12}$ IEL defects in normal BAs were frequently located near the VA junction and the BA bifurcation.

\section{Histology of the Dissected VA}

Our series included two victims with VA dissection. One of these victims was a 55-year-old man who had no relevant past medical history. He was the driver of a car that had fallen into a river. He was found dead in the car, and a legal autopsy was performed approximately 6 hours after death. The cause of death was drowning. There were no gross VA abnor- 

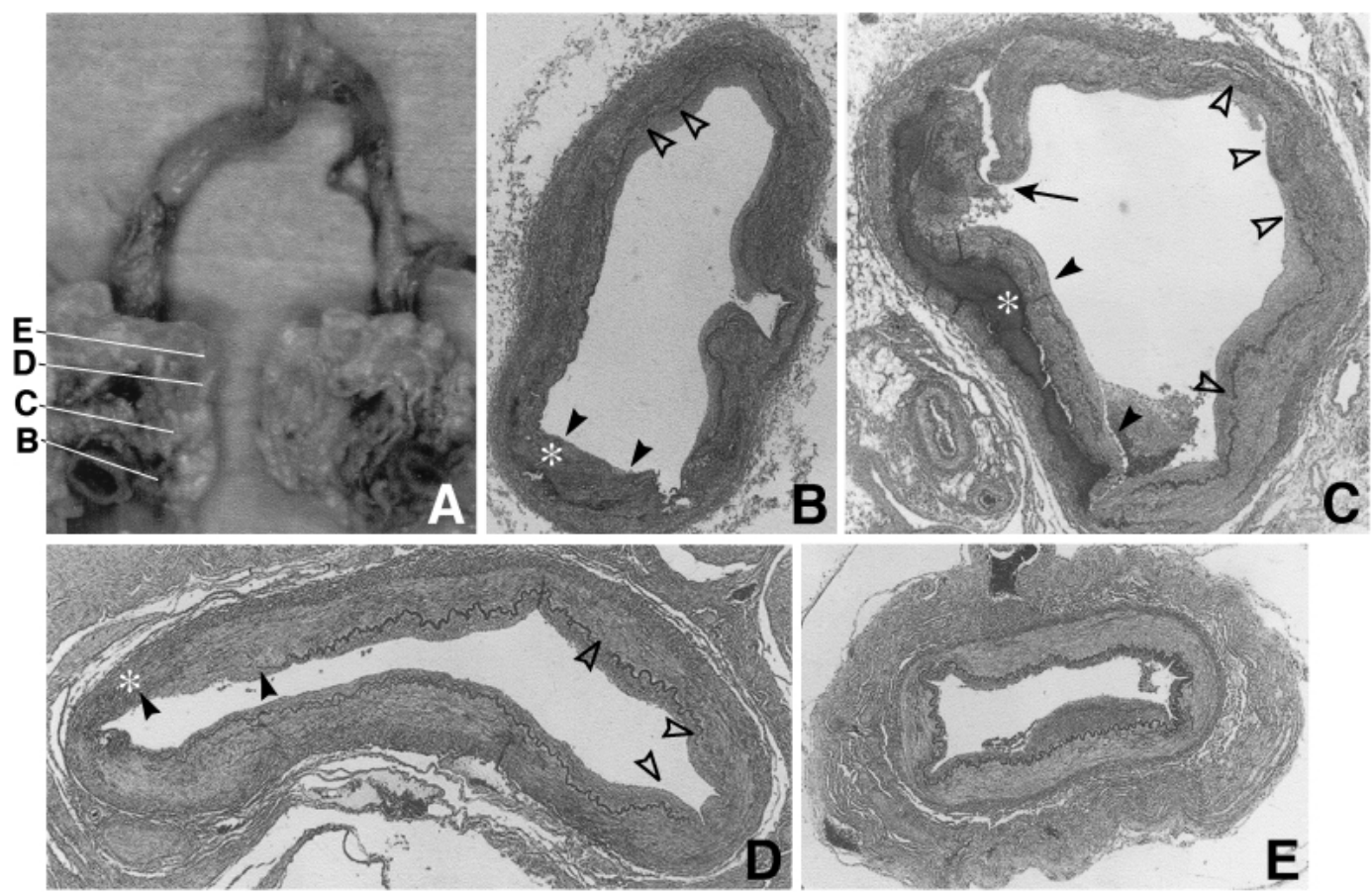

Fig. 5 A 55-year-old man. A: There were no gross abnormalities in the vertebral artery (VA). B-E: Sections from vascular blocks of the right VA showing dissection extending from $B$ to $D$ with an opening at C. Defects of the internal elastic lamina (IEL) were observed at 17 sites in this case. Cross-sections of the dissected lumen showed many extensive defects of the IEL at sites distant from the site of dissection. Other cross-sections showed defects of the IEL without marked thickening of the intima. Modified Masson's trichrome staining for elastic fibers, $\times 80$. asterisk: Dissected lumen, closed arrowheads: defects of the IEL near the dissected lumen, open arrowheads: defects of the IEL far from the dissected lumen, arrow: rupture point.

malities (Fig. 5A). Vascular blocks were prepared at 5-mm intervals, and sections from each block were examined under a light microscope. Dissection with an opening was noted in the right VA (Fig. 5B-D). Fibrin was attached to the lumen of dissection; however, as no collagen fiber proliferation was noted, the dissection was considered to be new. IEL defects were present at 17 sites in the bilateral VAs. In cross-sections showing the dissected lumen, there were many extensive IEL defects at sites distant from the dissected lumen. In other cross-sections, we also noted IEL defects; there was little intimal thickening at these sites.

Another victim with dissection of the VA was a 37-year-old man with a history of hypertension but no history of trauma. He was transported to our hospital complaining of sudden onset of pain in the head and neck and vomiting. His consciousness was clear at admission and no obvious motor paralysis was noted. Computed tomography showed diffuse $\mathrm{SAH}$, and angiography revealed "pearl and string" sign in the right VA at the origin of and distal from the PICA (Fig. 6A). The diagnosis was dissecting aneurysm of the right VA and surgery was performed. The VA distal to the branching-off of the PICA was clipped and the dissected lumen on the heart side of the branching-off of the PICA was clamped with two fenestrated clips (Fig. 6B). However, he died 5 days after surgery due to rebleeding. Autopsy findings were rupture of the IEL, media, and adventitia of the VA at point $F$ on the heart side of the branching-off of the PICA. Fibrin deposits and neutrophil infiltration were observed in the vascular wall around the site of dissection, suggesting that some time had lapsed since the occurrence of dissection and that the initial hemorrhage had occurred at onset near point F. There was an intramural hematoma between the media and adventitia on the heart side of the dissection site, and part of the adventitia of the hematoma lumen had ruptured (Fig. 6F). The hematoma near the dissection site showed fresh pathological features and death was thought to be due to hemorrhage from the dissection site. All components of the 


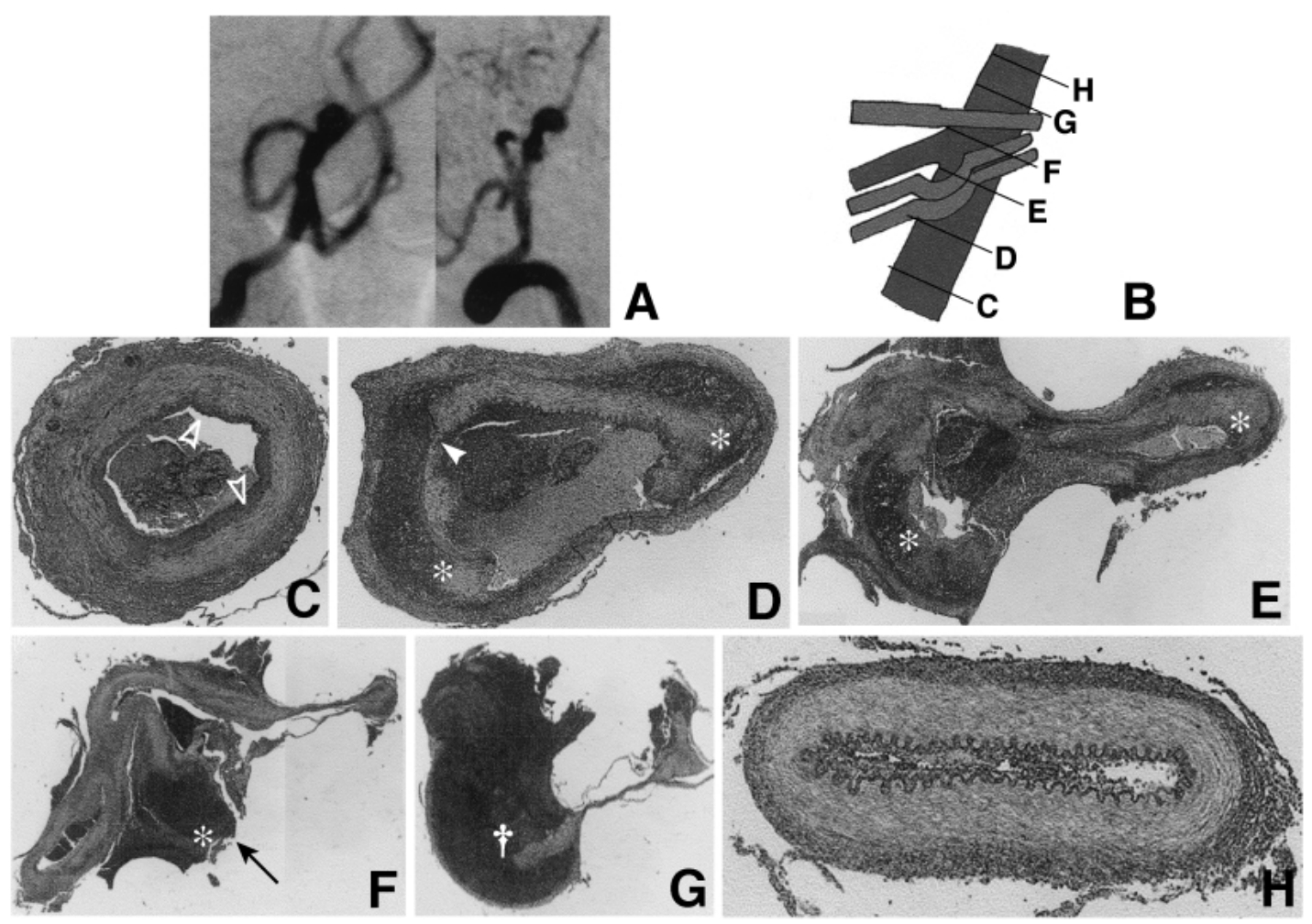

Fig. 6 A 37-year-old man. A: Vertebral angiograms showing pearl and string sign in the right vertebral artery (VA). B: VA distal to the division of the posterior inferior cerebellar artery (PICA) was obstructed by clipping, and the dissected lumen on the heart side of the PICA division was clamped with two fenestrated clips. C-H: Histological examination showed rupture of the internal elastic lamina (IEL), media, and adventitia of the VA on the heart side of the PICA division. Modified Masson's trichrome staining for elastic fibers, $\times 160$. asterisk: Dissected lumen, dagger: thrombosed lumen, closed arrowhead: defect of the IEL near the dissected lumen, open arrowheads: defects of the IEL far from the dissected lumen, arrow: rupture point.

vascular wall near the entry of the dissected lumen had been lost at rupture, and no evidence for the cause of the dissection could be obtained. IEL defects were noted at point $\mathrm{D}$ around the dissected lumen, the IEL was absent sporadically in the contralateral VA, and six defects were detected in this case.

\section{Discussion}

It is currently accepted in the field of legal medicine that neither predisposition nor physiologic changes due to aging should be taken into consideration in damage claims. For example, if an aged person with unsteady gait falls down due to slight contact with a pedestrian and sustains a head injury, the victim is not responsible for the unsteady gait.

IEL defects frequently occur with aging. If someone causes an accident that results in vascular injury in a victim, there is legal responsibility even if the victim had a pre-existing IEL defect. On the other hand, proportional judgment of the causal relationship is now widely accepted. According to this concept, if a person with a pre-existing arterial aneurysm or dissection suffers arterial rupture as a consequence of the action of an accidental external force, this is considered a mitigating factor.

A case reported by Schuster et al. ${ }^{13)}$ is instructive. A 16-year-old boy who was struck in the back of the head by a baseball lost consciousness for 5 minutes, regained consciousness for a short period, and then became comatose. On admission, his Glasgow Coma Scale score was 3. Computed tomography demonstrated SAH with intraventricular hemorrhage. The initial arteriogram showed no evidence of vascular abnormality. Because of the suspicious nature of his $\mathrm{SAH}$, another angiogram was obtained 72 hours after the injury, which revealed a 5-mm aneurysm 
arising from the origin of the right PICA.

This is an illustration of non-accountability for the victim's pre-existing diathesis. However, if the initial angiogram had not been obtained and the angiographic study performed 72 hours after the accident had revealed an aneurysm, what conclusion should be drawn?

The assessment of causal relationships in accident victims with head injuries is important in both civil and criminal cases. It is very difficult to judge causal relationship if there is a time lag between the time of the accident and the rupture or occlusion of a dissected artery. The risk of vascular rupture or dissection by accidental delivery of an external force to the head or face of victims must be considered in the assessment of causal relationships.

\section{Acknowledgments}

This study was supported in part by Grant in Aid for Scientific Research (C) (2) (No. 11670422) from the Japan Society for the Promotion of Science.

\section{References}

1) Bratzke H: Zur Morphologie der traumatischen Hirnstammschaedigung (biomechanische und differentialdiagnostische Aspekte). Zentralbl Rechtsmed 26: 205-222, 1984 (Ger)

2) Dolman CL: Rupture of posterior inferior cerebellar artery by single blow to head. Arch Pathol Lab Med 110: 494-496, 1986

3) Ezzat W, Ang LC, Nyssen J: Pontomedullary rent. A specific type of primary brainstem traumatic injury. Am J Forensic Med Pathol 16: 336-339, 1995

4) Fraenckel P: Gedeckte traumatische Xerreissung der gesunden Arteria basilaris. Dtsch Z Ges Gerichtl Med 10: 192-199, 1927 (Ger)

5) Gunji H, Mizusawa I, Hiraiwa K: The mechanism underlying the occurrence of traumatic brainstem lesions in victims of traffic accidents. Leg Med (Tokyo) 4: 84-89, 2002

6) Haldeman S, Kohlbeck FJ, McGregor M: Risk factors and precipitating neck movements causing vertebrobasilar artery dissection after cervical trauma and spinal manipulation. Spine 24: 785-794, 1999

7) Hiraiwa K, Mizusawa I, Gunji H, Sato T: [Mechanism of traumatic vertebrobasilar artery rupture]. Shinkeigaisho 23: 32-36, 2000 (Jpn)

8) Johnson CP, Lawler W, Burns J: Use of histomorphometry in the assessment of fatal vertebral artery dissection. J Clin Pathol 46: 1000-1003, 1993

9) Kondo T, Saito K, Nishigami J, Ohshima T: Fatal injuries of the brain stem and/or upper cervical spinal cord in traffic accidents: nine autopsy cases. Sci Justice 35: 197-201, 1995

10) Lindenberg R, Freytag E: Brainstem lesions characteristic of traumatic hyperextension of the head. Arch Pathol 90: 509-515, 1970

11) Marek Z: Isolated subarachnoid hemorrhage as a medicolegal problem. Am J Forensic Med Pathol 2: 19-22, 1981

12) Sato T, Sasaki T, Suzuki K, Matsumoto M, Kodama N, Hiraiwa K: Histological study of the normal vertebral artery - etiology of dissecting aneurysms. Neurol Med Chir (Tokyo) 44: 629-636, 2004

13) Schuster JM, Santiago P, Elliott JP, Grady MS, Newell DW, Winn HR: Acute traumatic posteroinferior cerebellar artery aneurysms: report of three cases. Neurosurgery 45: 1465-1468, 1999

14) Simpson DA, Blumbergs PC, Cooter RD, Kilminster M, McLean AJ, Scott G: Pontomedullary tears and other gross brainstem injuries after vehicular accidents. J Trauma 29: 1519-1525, 1989

15) Voigt GE, Skold G: Ring fractures of the base of the skull. J Trauma 14: 494-505, 1974

16) Wilkinson IMS: The vertebral artery. Extracranial and intracranial structure. Arch Neurol 27: 392-396, 1972

17) Wolff K: Traumatische Zerreissung der gesunden Art. vertebralis an der Hirnbasis. Dtsch Z Ges Gerichtl Med 10: 464-467, 1928 (Ger)

18) Yamaura A: Nontraumatic intracranial arterial dissection. Natural history, diagnosis, and treatment. Contemporary Neurosurgery 16: 1-6, 1994

Address reprint requests to: K. Hiraiwa, M.D., Department of Legal Medicine, Fukushima Medical University, 1 Hikarigaoka, Fukushima 960-1295, Japan. e-mail: khiraiwa@fmu.ac.jp 